\title{
Characterization and functional analysis of microRNA399 in Cunninghamia lanceolata
}

\author{
F.R. ZHU' ${ }^{1}$, Z.B. QIU ${ }^{2 *}$, Y.M. ZHANG ${ }^{2}$, X. R. ZHANG ${ }^{2}$, and W. L.WANG ${ }^{2}$ \\ School of Life Science and Technology, Xinxiang University, Xinxiang 453003, P.R. China ${ }^{1}$ \\ College of Life Science, Henan Normal University, Xinxiang 453007, P.R. China ${ }^{2}$
}

\begin{abstract}
The miR399 is a conserved microRNA (miRNA) family, and it has been characterized as an essential regulator of phosphorus transport in plants. However, the biological function of miR399 in Cunninghamia lanceolata is still largely unclear. In this study, the comparison of mature miR399 sequence revealed a high similarity between Arabidopsis thaliana and C. lanceolate, and the pre-miR399 was capable of forming a typical stem-loop hairpin structure. A gene PHOSPHATE 2 (PHO2) was identified as a target of $c l n$-miR399 using 5' rapid amplification of cDNA ends. Furthermore, the relationship between $c \ln$-miR399 and PHO2 was further confirmed through a transient co-expression of both genes in Nicotiana benthamiana. To examine the function of miR399 in Arabidopsis, miR399-overexpressing transgenic Arabidopsis thaliana was acquired using Agrobacterium-mediated approach. Real-time PCR showed that the amount of $c l n$-MIR399 transcripts was higher in miR399-overexpressing plants than in wild-type plants, which was accompanied with down-regulation of expression of its target gene AtPHO2. The $\mathrm{P}$ content was 1.40 to 1.56 -fold higher in the leaves of three transgenic lines than in wild type plants. However, the P content in the roots of the three transgenic lines was $24.5-37.2 \%$ less than that in wild type plants. Moreover, the transcriptions of three phosphate transporter genes (PHT1, PHT2, and PHT3) were up-regulated in roots of miR399-overexpressing Arabidopsis plants. Interestingly, the transgenic lines exhibited retarded growth under normal $\mathrm{P}$ conditions compared with the wild type. Our findings demonstrate that $c \ln$-miR399 may play crucial roles in P transport and plant growth via regulation of its target gene PHO2.
\end{abstract}

Additional key words: Arabidopsis thaliana, Nicotiana benthamiana, PHO2, phosphate transporters, RLM-RACE.

\section{Introduction}

MicroRNAs (miRNAs) represent a class of $20-24$ nucleotide (nt) non-coding small RNAs, which can regulate target mRNAs via directing cleavage or translational repression (Voinnet 2009, Cuperus et al. 2011). An increasing number of evidence indicates that miRNAs play critical roles in leaf morphogenesis, phase transitions, nutrient homeostasis, and various biotic or abiotic stress responses (Qiu et al. 2013, 2016, Hu et al. 2015, Hai et al. 2018).

The microRNA399 (miR399) is one of the most ancient and highly conserved microRNA families in monocots and dicots (Cuperus et al. 2011). Recently, miR399 has been demonstrated to target the PHOSPHATE 2 (PHO2) gene, which has been confirmed by using a modified 5 '-rapid amplification of cDNA ends (RACE) method. The $\mathrm{PHO} 2$ encodes an ubiquitin-conjugating E2 enzyme involved in ubiquitin-mediated protein degradation. In Arabidopsis and rice, miR399 has been confirmed to act as an important regulator of $\mathrm{P}$ acquisition and $\mathrm{P}$ metabolism through the downregulation of $\mathrm{PHO} 2$ transcription (Bari et al. 2006, Chiou et al. 2006), and rice transgenic lines with miR399 overexpression exhibit multiple nutrient starvation responses ( $\mathrm{Hu}$ et al. 2015). Furthermore, the overexpression of miR399 in transgenic plants could increase the content of sugars and vitamin $\mathrm{C}$, thus improving the quality of strawberry fruits (Wang et al. 2017). These findings suggest that miR399 acts mainly via down-regulating the target gene $\mathrm{PHO} 2$ to modulate a wide range of metabolic and other biological processes.

Chinese fir (Cunninghamia lanceolata Lamb. Hook) is one of the most important coniferous evergreen tree species due to its rapid growth, and thus it serves as a global resource of wood (Shi et al. 2010, Wan et al. 2012). A genome-scale analysis of miRNA expression

Submitted 23 April 2019, last revision 18 October 2019, accepted 8 January 2020.

Abbreviations: Pi - inorganic phosphorus; miRNA - microRNA; nt - nucleotide; PHO2 - PHOSPHATE 2; RACE - 5' rapid amplification of cDNA ends; RLM - RNA ligase-mediated.

Ackowledgements: This work was supported by the National Natural Science Foundation of China (31500499) and the Program for Science Technology Innovation Talents in Universities of Henan Province (16HASTIT019).

* Corresponding author; e-mail: qiuzongbo@126.com 
profiling in $C$. lanceolata was carried out by using highthroughput sequencing, and 20 known miRNAs families including miR156, miR159, miR172, miR396, miR399, and miR408 have been identified in our previous study (Wan et al. 2012, Qiu et al. 2015). Although more and more studies have demonstrated the critical roles for miR399 and its targets PHO2 in Arabidopsis, tomato, and strawberry (Bari et al. 2006, Gao et al. 2015, Wang et al. 2017); relatively less is known about the function of miR399 and its targets in C. lanceolata. To evaluation the roles of miR399 in mediating P transport and plant growth, transgenic Arabidopsis with cln-MIR399 overexpression were generated using Agrobacterium-mediated approach. Furthermore, the expression of the miR399 precursor, PHO2 mRNA, as well as inorganic phosphorus (Pi) content were analyzed in wild-type and miR399-overexpressing Arabidopsis. Subsequently, the phenotypes of transgenic Arabidopsis ectopically expressing cln-miR399 were characterized. These results could deliver a new insight into the function of cln-miR399 and its target and could provide the basis for further functional studies of miRNA in conifers.

\section{Materials and methods}

Plants and growth conditions: Arabidopsis thaliana L. ecotype Columbia (Col-0) was used to obtain transgenic lines. The wild type, transgenic Arabidopsis plants and Nicotiana benthamiana Domin seedlings were grown in a greenhouse at a temperature of $22^{\circ} \mathrm{C}$, a 16-h photoperiod, an irradiance of $120 \mu \mathrm{mol} \mathrm{m} \mathrm{m}^{-2} \mathrm{~s}^{-1}$, and a realtive humidity of $70 \%$. Seeds of Chinese fir (Cunninghamia lanceolata Lamb. Hook) were obtained from the Fujian Academy of Forest Sciences, Fujian, China and small seedlings were grown under above mentioned conditions. The seedlings (7-d-old) of wild type and transgenic Arabidopsis plants showing consistent growth were transplanted into soil supplemented with a one-half Hoagland nutrient solution containing $250 \mu \mathrm{M} \mathrm{KH} \mathrm{KH}_{2} \mathrm{PO}_{4}$ (the normal phosphorus concentration).

Sequence analysis: Precursor sequences of miR399 in C. lanceolata were examined for the secondary structure using the Mfold program with the default parameters (Zuker 2003). Mature miR399 sequences of Arabidopsis were downloaded from miRBase release 21.0 (http:// www.mirbase.org). Multiple sequence alignments of $c l n$ miR399 and Arabidopsis miR399a-f mature sequences were performed using the DNAMAN software.

To define target genes of $c \ln$-miR399, the mature sequence of $c \ln$-miR399 was used as a query to search against the Chinese fir mRNA transcriptome database (59 669 sequences) using the web-based program psRNATarget with default parameters (Zhang 2005). The sequence of PHO2 (a ubiquitin-conjugating E2 enzyme) was obtained from Arabidopsis thaliana (TAIR v. 10).

Rapid amplification of cDNA ends: A modified RNA ligase-mediated 5' rapid amplification of cDNA ends (RLM-RACE) was conducted to obtain cleavage transcripts based on the method described by Ding et al. (2016). Gene-specific primers are listed in Table 1 Suppl.

Transient co-expressions of cln-MIR399 and PHO2: The sequence of the miR399 precursor was amplified from the genomic DNA in three-month-old C. lanceolata seedlings with the following primer pairs: forward, 5'-GGGGATAATTACTGGGGGACTCTTC-3'; reverse, 5'-AGAACAATTGCAGGGCAACTCTCCT-3'. The amplified fragment was introduced into a binary vector (pCAMBIA2300) under the control of the cauliflower mosaic virus $35 \mathrm{~S}$ promoter. The target gene $\mathrm{PHO} 2$ was amplified from Arabidopsis or C. lanceolata with primers listed in Table 1 Suppl. and inserted into the same vector. Agrobacterium suspensions harboring cln-MIR399 and $\mathrm{PHO} 2$ were infiltrated separately or coinfiltrated into the leaves of 4-week-old tobacco as previously described (Zheng et al. 2012). For co-expression of $\ln$-MIR399 and PHO2, two kinds of Agrobacterium suspensions were mixed at a 1:1 ratio before infiltration of tobacco leaves.

Cloning and overexpression of cln-MIR399 in Arabidopsis: To overexpress $\operatorname{cln}$-MIR399 in Arabidopsis, pre-miR399 was cloned via reverse transcription PCR amplification using primers 5'-GGGGATAATTACTGGGGGACTCTTC-3' and 5'-AGAACAATTGCAGGGCAACTCTCCT-3'. The amplified fragments were sequenced and then subcloned into the binary vector pCAMBIA2300 between the KpnI and BamHI sites to generate a 35S:MIR399 construct. The construct containing the $35 \mathrm{~S}$ promoter was transformed into the Agrobacterium tumefaciens strain EHA105 and was then transferred into wild-type Col-0 ecotype plants using the method proposed by Bechtold and Pelletier (1998). Transgenic seeds were screened on a medium containing kanamycin and validated by PCR amplification. Subsequently, $\mathrm{T}_{3}$ homozygous lines were used for phenotypic characterization and gene expression analysis.

Real-time quantitative PCR: Total RNA isolation, cDNA synthesis from the total RNA, and followed real-time quantitative PCR analysis were conducted according to Qiu et al. (2016) and Hai et al. (2018). For amplification, specific primers were designed for $c \ln$-MIR399, AtPHO2, PHT1, PHT2, and PHT3 (Table 1 Suppl.). Relative expressions of different genes were normalized against an internal reference gene Arabidopsis tubulin using the $2^{-\Delta \Delta \mathrm{Ct}}$ method (Livak and Schmittgen 2001).

Determination of total P content: Roots and shoots from 7-week-old wild-type plants and MIR399-overexpressing transgenic Arabidopsis lines were harvested and immediately frozen in liquid nitrogen. Total $\mathrm{P}$ content was determined according to the method proposed by $\mathrm{Hu}$ et al. (2015). Briefly, the leaves and roots were dried at $80{ }^{\circ} \mathrm{C}$ to a constant weight. Dried samples $(50 \mathrm{mg})$ were digested with $13 \mathrm{~cm}^{3}$ of concentrated $\mathrm{HNO}_{3}$ and $2 \mathrm{~cm}^{3}$ of $30 \% \mathrm{H}_{2} \mathrm{O}_{2}$ at $140{ }^{\circ} \mathrm{C}$ for $30 \mathrm{~min}$. The digested solutions were adjusted to a volume of $25 \mathrm{~cm}^{3}$ with de-ionized water. The metal 
elements were determined using inductively coupled plasma optical emission spectrometry (PE, Optima 2000 $D V$, Waltham, MA, USA).

Statistics: All samples were carried out in three biological triplicates, and results were represented as means \pm SEs of three replicates. For evaluation of significant differences at $\alpha=0.05$, the Duncan's multiple range test was used.

\section{Results}

The miR399 family belongs to conserved miRNA families across diverse plant species, and $c \ln$-miR399 has been identified from $C$. lanceolata in our previous study based on high-throughput sequencing (Qiu et al. 2015). Mature sequences of Arabidopsis miR399 family members were derived from miRBase release 21 . The alignment of mature sequences in C. lanceolata miR399 and Arabidopsis miR399a-f were conducted using the multiple sequence alignment method. The results show that the mature sequence of $c \ln$-miR399, which was 21-nt long (5'-UGCCAAAGGAGAGUUGCCCUG-3'), was the same as Arabidopsis mature miR399b and miR399-3p sequences (Fig. 1) indicating that the miR399 family sequence was deeply conserved. The precursor of $c l n$ miR399 was amplified by PCR, and the 105 bp precursor sequence was capable of forming a stable stem-loop secondary structure (Fig. 1 Suppl.). The minimum folding free energy index of the pre-miR399 hairpin structure was 0.98 , and the average $\mathrm{A}+\mathrm{U}$ content of the premiR399 sequence was 58.1 (Table 1). As shown in Table 2, two potential target genes, PHOSPHATE 2 (PHO2) (unigene56556) and predicated protein (unigene84522)

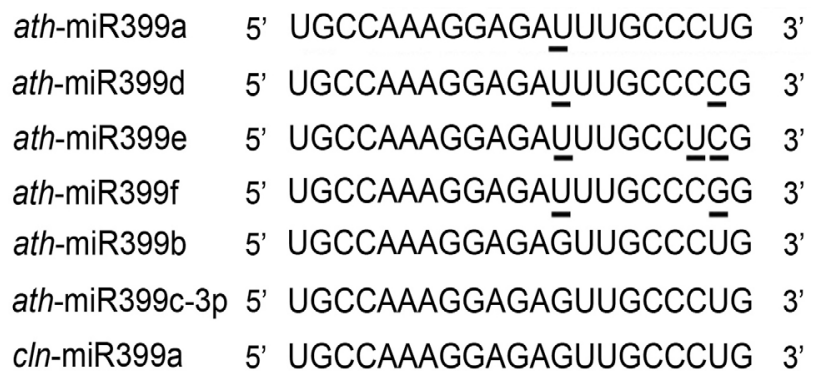

Fig. 1. Alignment of mature sequences in Cunninghamia lanceolata miR399 (cln-miR399a) and Arabidopsis miR399 (ath-miR399a-f). Mismatches are underlined.

were predicted from the $C$. lanceolata mRNA transcriptome database based on psRATarget analysis.

To evaluate whether miR399 could mediate the cleavage of putative target mRNA in C. lanceolata, we detected the cleavage products of unigene 56556 mRNA in 3-month-old $C$. lanceolata seedlings using 5'-RACE. The unigene 56556 encoding $\mathrm{PHO} 2$ had a cleavage site at the $10^{\text {th }}$ and $11^{\text {th }}$ nucleotide of miR399 from the $5^{\prime}$-end (Fig. 2). The cleavage event was further verified by Agrobacteriummediated transient co-expression in $N$. benthamiana. The results of transient co-expression demonstrate that the amount of PHO2 transcripts markedly decreased by $\mathrm{cln}$ miR399 (Fig. 3). These results provide direct evidence that the $\mathrm{PHO} 2$ gene is the true target of miR399 in Chinese fir.

To explore the function of $C$. lanceolata miR399, we generated transgenic Arabidopsis plants overexpressing cln-miR399 driven by the enhanced cauliflower mosaic virus $35 \mathrm{~S}$ promoter. Three independent transgenic lines were selected for further analysis. To further determine the

Table 1. Characteristics of a miR399 precursor from Chinese fir. MFE - minimum folding free energy, MFEI - minimal folding free energy index.

\begin{tabular}{lc}
\hline miRNA & cln-miR399 \\
\hline Mature sequence & UGCCAAAGGAGAGUUGCCCUG \\
Mature miRNA length [nt] & 21 \\
Precursor sequence & GGGGAUAAUUACUGGGGGACUCUUCUUUGGCUGGAAUUAUUCAAUCUCUUAAC \\
& UCAUGUUAAUGUUUUGUUGCCUGCCAAAGGAGAGUUGCCCUGCAAUUGUUCU \\
Pre-miRNA length [nt] & 105 \\
Arm location & 3 \\
MFE [kJ mol-1] & -43.20 \\
A+U [\%] & 58.1 \\
MFEI & 0.98 \\
\hline
\end{tabular}

Table 2. Predicted target genes for miR399 and their putative functions.

\begin{tabular}{lllll}
\hline miRNA & Target genes & Score & Predicted function & GO annotation \\
\hline cln-miR399 & unigene 56556 & 0.5 & phosphate 2 & phosphate homeostasis \\
& unigene84522 & 0.5 & predicated protein & \\
\hline
\end{tabular}




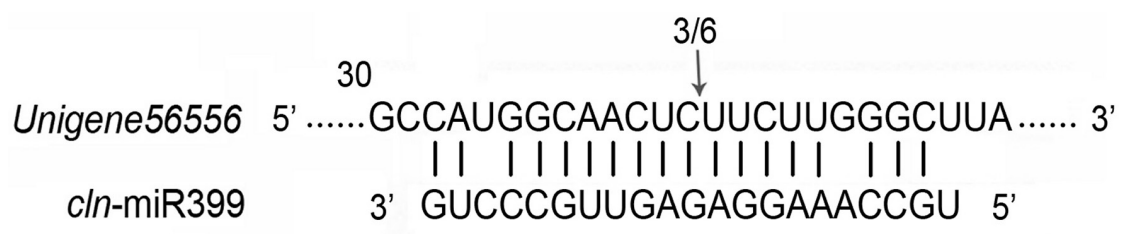

Fig. 2. The 5' rapid amplification of cDNA ends of miRNA cleavage sites: the miRNA sequence (bottom) and its corresponding target mRNA sequence (top) is shown in each alignment. The arrow indicates a cleavage site detected by RNA ligase-mediated 5' rapid amplification of cDNA ends, and the number above the arrow indicates the number of cleaved/total clones sequenced.
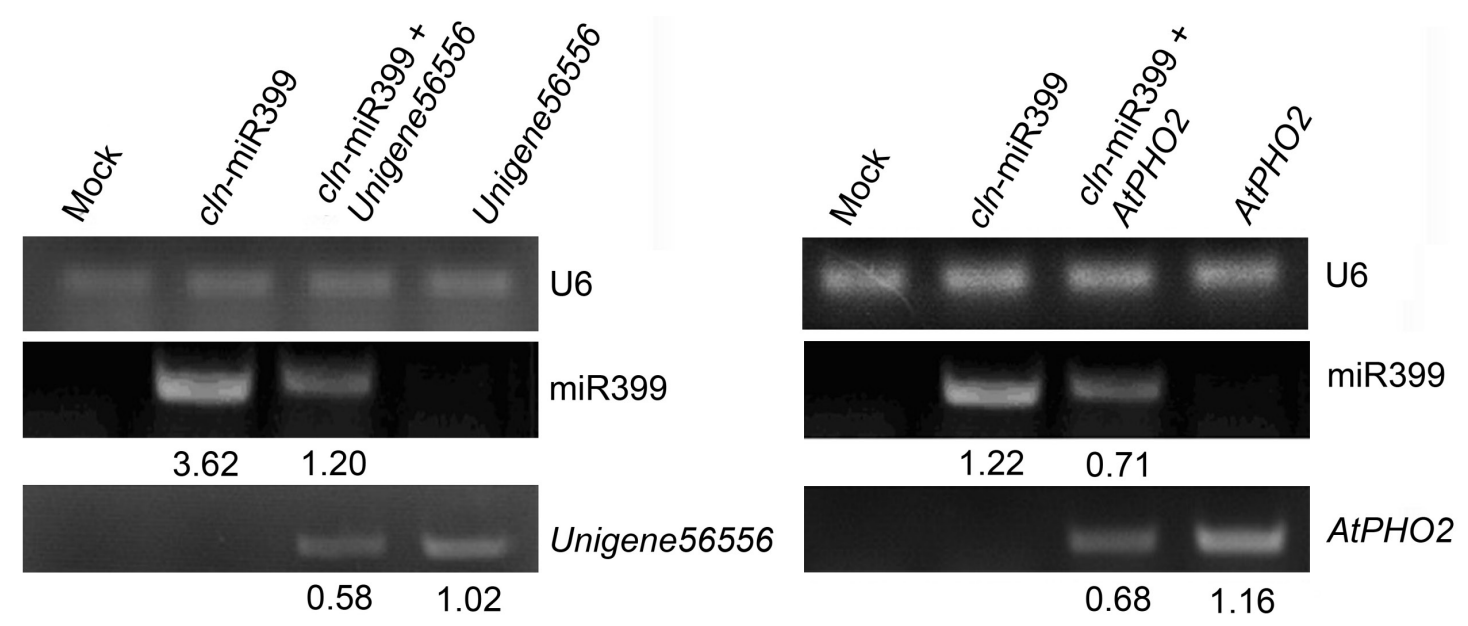

Fig. 3. Transient co-expression assay of Cunninghamia lanceolata cln-miR399 $(A, B)$ and $C$. lanceolata unigene $56556(A)$ and Arabidopsis PHO2 (B) in Nicotiana benthamiana leaves. Accumulations of mRNA of $c \ln$-miR399, unigene56556, and PHO2 are shown. Leaves transformed with an empty vector served as a loading control (Mock).

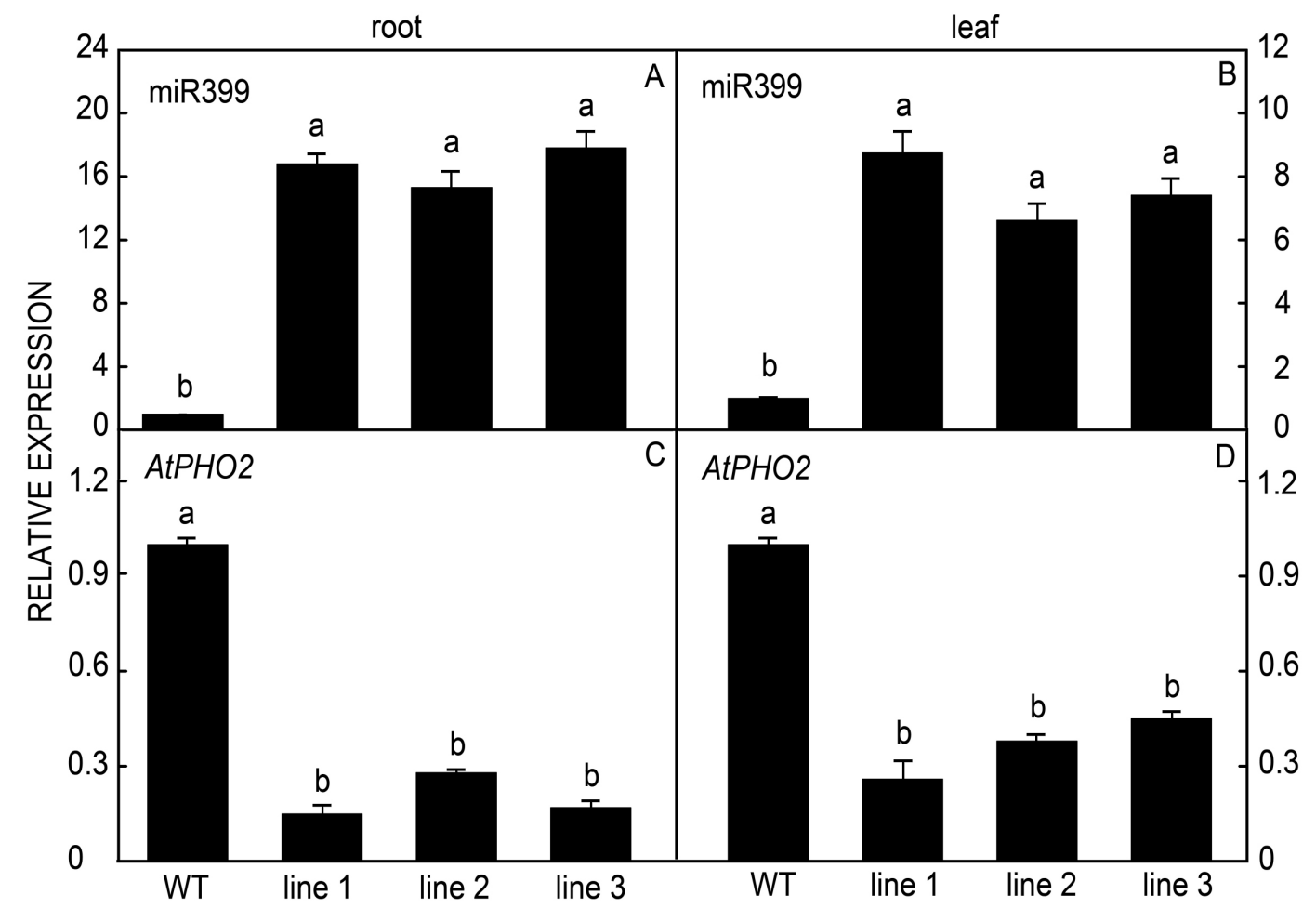

Fig. 4. Expression analysis of $c \ln$-MIR399 $(A, C)$ and its target $P H O 2(B, D)$ in the roots and leaves of wild type (WT) and transgenic lines 1, 2, and 3 under normal P conditions. The expressions of miR399 and PHO2 in the WT were set to 1 . Means \pm SEs, $n=3$, different letters indicate significant differences according to the Duncan's multiple range test at $P \leq 0.05$. 


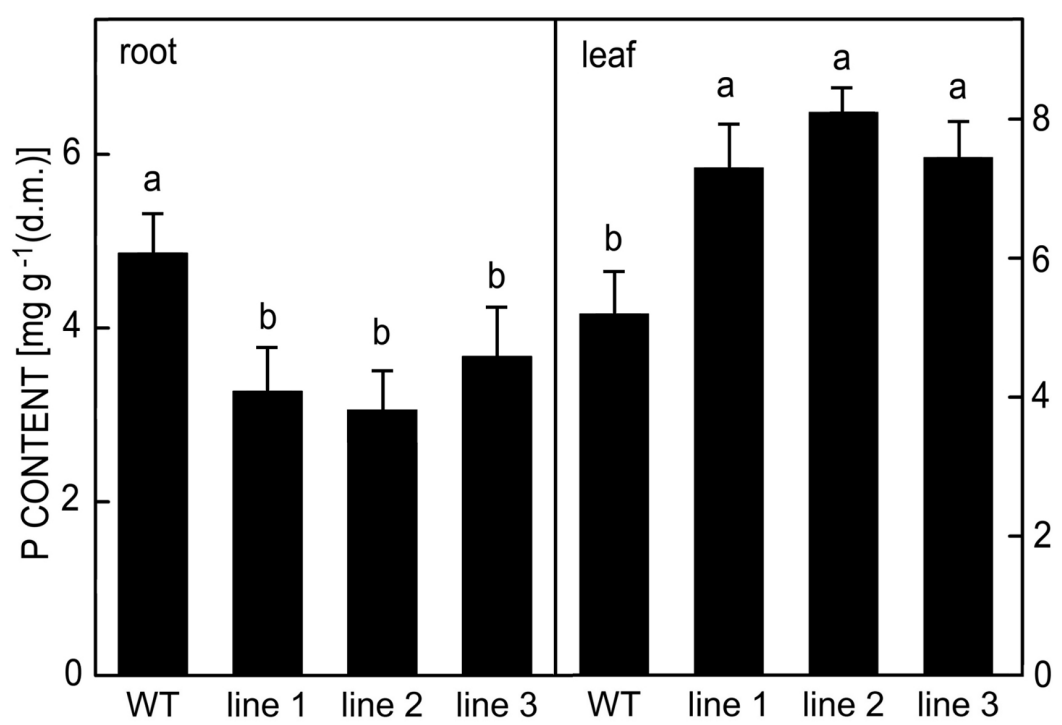

Fig. 5. Phosporus content in leaves and roots of seven-week-old wild-type and transgenic Arabidopsis lines grown at normal P conditions. Means \pm SEs, $n=3$; different letters indicate significant differences according to the Duncan's multiple range test at $P \leq 0.05$.

expression of $c \ln$-MIR399 in the three lines, total RNA was isolated from 4-week-old wild type Arabidopsis plants and transgenic lines and analyzed by real-time quatitative PCR. As shown in Fig. 4, the transcription of $c l n$-MIR399 in the three independent transgenic lines was much higher than in the wild-type plants. Interestingly, $c \ln$-MIR399 was also more expressed in roots of transgenic plants than in their leaves. A high miR399 accumulation in transgenic plants suggests that $c \ln$-MIR399 was successfully expressed in Arabidopsis. By contrast, the target gene PHO2 transcriptions were substantially lower in the roots and leaves of the three transgenic lines exhibiting an opposite trend of expression to cln-MIR399 suggesting that the PHO2 mRNA abundance could be negatively regulated by miR399. To further examine a relationship between cln-MIR399 and AtPHO2, Agrobacterium-mediated transient co-expressions of $\operatorname{cln}$-MIR399 and AtPHO2

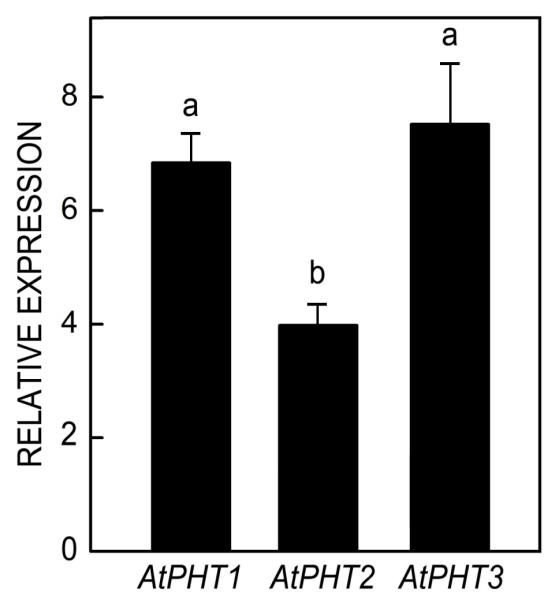

Fig. 6. Transcript abundances of three phosphate transporter genes in transgenic Arabidopsis lines under normal P conditions. The expression in the wild type was set to 1 . Means \pm SEs, $n=3$. in $N$. benthamiana were established. The results of the transient co-expressions show that AtPHO2 markedly decreased by $c$ n-MIR399 (Fig. 3) suggesting that miR399 could directly cleavage and degrade mRNA of $\mathrm{PHO}$.

To assess whether miR399 overexpression affected $\mathrm{P}$ transport and plant growth, we grew seven-week-old wild type Arabidopsis plants and miR399-overexpressing Arabidopsis lines at normal $\mathrm{P}$ conditions in soil for $5 \mathrm{~d}$. After $5 \mathrm{~d}$, we measured the total $\mathrm{P}$ content in transgenic lines and wild-type plants. The $\mathrm{P}$ content increased 1.40 to 1.56 -fold in the leaves of the three transgenic lines compared with the wild-type plants. However, the P content in the roots of the three transgenic lines was 24.5 to $37.2 \%$ less than that in the wild-type plants (Fig. 5).

To estimate the effect of miR399 on Pi transport and accumulation in leaves, we next used real-time quantitative PCR to analyze the expressions of the three phosphate transporter (PHT) genes in transgenic Arabidopsis plants and wild-type plants. As a result, relative expressions of AtPHT1, AtPHT2, and AtPHT3 increased more in roots of transgenic lines in comparison with wild-type plants at normal $\mathrm{P}$ conditions (Fig. 6) indicating that they were positively regulated by miR399 and important for $\mathrm{Pi}$ transport and accumulation in Arabidopsis.

To investigate the effect of the miR399 overexpression on growth of the plants, we characterized the phenotype of the miR399-overexpressing plants. As shown in Fig. 7, two-week-old transgenic plants displayed shorter roots than wild type plants. Furthermore, compared with the wild type, seven-week-old transgenic plants exhibited a retarded growth.

\section{Discussion}

The miR399 is a conserved miRNA family existing in several plant species, and this family is predicted to target 


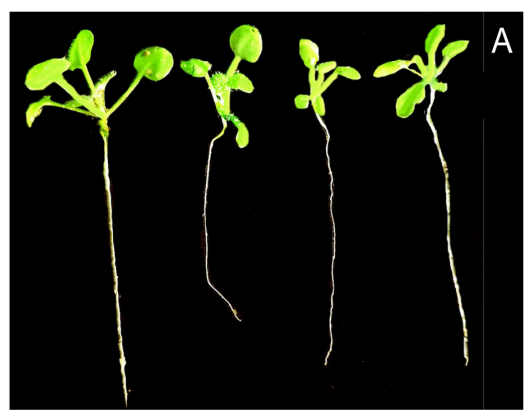

WT line 1 line 2 line 3

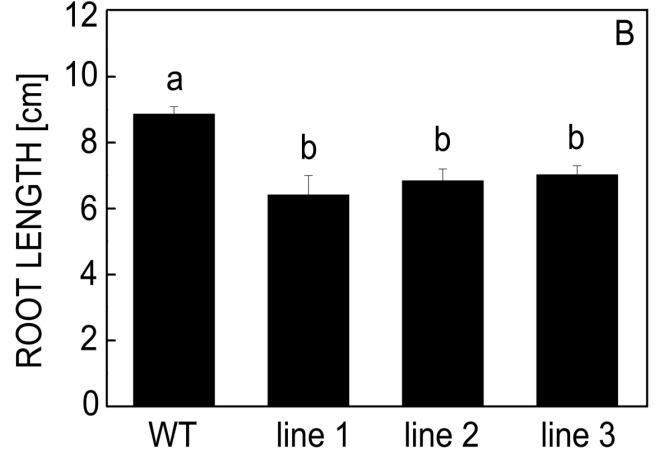

WT

line 1

line 2

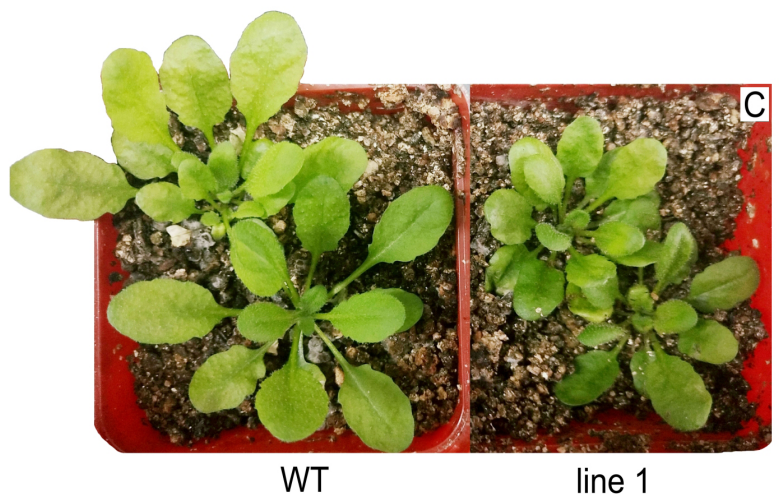

Fig. 7. Growth features of wild type and transgenic Arabidopsis plants grown under normal $\mathrm{P}$ conditions in soil determined two $(A)$ and seven $(C)$ weeks after seed germination. $B$ - Root length of wild type (WT) and transgenic Arabidopsis plants. Means \pm SEs, $n=15$. Different letters indicate significant differences according to the Duncan's multiple range test at $P \leq 0.05$.

PHO2 (Lin et al. 2008, Hackenberg et al. 2013). In the present investigation, we searched for putative target genes for $c \ln$-miR399 by bioinformatics prediction and identified two targets, $\mathrm{PHO} 2$ (unigene 56556) and predicated protein (unigene 84522) as a candidate target for miR399. Similar to that in $C$. lanceolata, miR399 also targets $\mathrm{PHO} 2$ genes in Arabidopsis, barley, and strawberry (Lin et al. 2008, Hackenberg et al. 2013, Wang et al. 2017). To test whether PHO2 is subjected to miRNA-mediated cleavage in vivo, we isolated mRNAs from three-month-old $C$. lanceolata seedlings and performed 5'-RACE method to detect the 3' cleavage products. A 5'-RACE assay is a simple and efficient method for in vivo assays of the cleavage sites of miRNAs on their mRNA targets. Here, PHO2, which encodes PHOSPHATE 2, was verified and characterized as the target gene of $c \ln$-miR399 by 5 '-RACE suggesting that the target identified by bioinformatics prediction is indeed authentic, and miR399 can target and cleave the corresponding transcripts in C. lanceolata.

To confirm whether Arabidopsis $\mathrm{PHO} 2$ transcripts could be directly cleaved by $c \ln$-MIR399, we investigated a relationship between $c \ln$-MIR399 and AtPHO2 through transient co-expressions of both genes in tobacco leaves. Interestingly, $c l n$-MIR399 expression markedly decreased when co-expressed with 35S::cln-MIR399. Our results are consistent with the results of Wang et al. (2010), who demonstrated that miR399 can cleave PHO2 mRNA in Arabidopsis and tobacco leaves. Our results show that Arabidopsis PHO2 was a target of $c \ln$-MIR399 and that $c \ln$-MIR399 had the capability to direct the cleavage of
AtPHO2 in vivo.

To further dissect the relationship between $\mathrm{cln}$ miR399 and its target PHO2, the transcriptions of miR399 and AtPHO2 were monitored in the 35S: cln-MIR399 transgenic plants and the wide-type plants by real-time quantitative PCR. The results show that miR399 was successfully up-regulated in $c \ln$-miR399-overexpressing plants as compared with wild type plants. Interestingly, cln-miR399 expression in the roots of transgenic plants was much higher than in the leaves. Our results are in agreement with a study in tomato, in which miR399 is highly expressed in roots of miR399-overexpressing plants (Gao et al. 2015). Additionally, the expression analysis revealed a significant down-regulation of miR399 target genes (AtPHO2) in both the roots and leaves of $c l n$ miR399-overexpressing plants in comparison with the wild type and exhibited a negative impact on the expression of miR399. Taken together, these results suggest that miR399 derived from a $C$. lanceolata precursor was heterologously expressed in Arabidopsis, and miR399 controlled a wide range of metabolic processes in $C$. lanceolata by a negative regulation of $\mathrm{PHO} 2$.

Inorganic phosphorus is one of the most significant mineral nutrients for plants as it constitutes many important biological molecules such as nucleic acids, phospholipids, and ATP. In soil solution, the concentration of Pi is very low, and consequently, P starvation often occurs. Recently, a new type of regulatory element, miR399, has been confirmed to act as a mediator in improving P uptake and translocation under P deficiency conditions in Arabidopsis 
and rice (Chiou et al. 2006, $\mathrm{Hu}$ et al. 2015). The line overexpressing miR399 results in $\mathrm{P}$ accumulation in shoots of Arabidopsis (Fujii et al. 2005), strawberry (Wang et al. 2017), and tomato (Gao et al. 2015). These results are in agreement with our results where overexpression of $c \ln$-miR399 led to increased P content in the leaves of Arabidopsis. However, the P content in the roots of transgenic lines was lower than that in the wild type. The lower P content in the roots of transgenic Arabidopsis is consistent with results obtained on transgenic woodland strawberry (Wang et al. 2017). These results suggest that the overexpression of miR399 could enhance P transfer ability and affect $\mathrm{P}$ distribution from roots to shoots. We next considered whether overaccumulation of $\mathrm{P}$ in the leaves resulted from an increased uptake of $P$. Therefore, transcriptions of AtPHT1, AtPHT2, and AtPHT3, which are important phosphate transporters controlling $\mathrm{P}$ uptake and translocation, were analyzed by real-time quantitative PCR. We found that the expression of AtPHT1, AtPHT2, and AtPHT3 more increased in the roots of transgenic lines than in the wild type at the normal $P$ conditions. These results are in agreement with that of Chiou et al. (2006), who showed that the expressions of two phosphate transporter genes, AtPHT1 and AtPHT2 in roots of miR399-overexpressing Arabidopsis are higher than in a wild type. Gao et al. (2015) also reported that overexpressing ath-miR399d enhances the transcriptions of phosphate transporters LePT1, LePT2, LePT4, and LePT5 in P-sufficient roots of transgenic tomato. Therefore, miR399 activated by downregulating $\mathrm{PHO} 2$ the expression of $\mathrm{P}$ transporters, such as AtPHT1 and AtPHT2, and consequently enhanced P uptake and transport to shoots.

In summary, this study demonstrated that miR399 derived from a $C$. lanceolata precursor together with its target gene $\mathrm{PHO} 2$ were confirmed to be critical in regulating $\mathrm{P}$ transport and plant growth. The precise mechanisms how cln-miR399 regulates the P transport and plant growth should be further elucidated.

\section{References}

Bari, R., Pant, B.D., Stitt, M., Scheible, W.: PHO2, microRNA399, and PHR1 define a phosphate-signaling pathway in plants. Plant Physiol. 141: 988-999, 2006.

Bechtold, N., Pelletier, G.: In planta Agrobacterium-mediated transformation of adult Arabidopsis thaliana plants by vacuum infiltration methods. - Mol. Biol. 82: 259-266, 1998.

Chiou, T.J., Aung, K., Lin, S.I., Wu, C.C., Chiang, S.F., Su, C.L.: Regulation of phosphate homeostasis by microRNA in Arabidopsis. - Plant Cell 18: 412-421, 2006.

Cuperus, J.T., Fahlgren, N., Carrington, J.C.: Evolution and functional diversification of miRNA genes. - Plant Cell 23: 431-442, 2011.

Ding, Q., Zeng, J., He, X.Q.: miR169 and its target PagHAP2-6 regulated by $\mathrm{ABA}$ are involved in poplar cambium dormancy.
- J. Plant Physiol. 198: 1-9, 2016.

Fujii, H., Chiou, T.J., Lin, S.I., Aung, K., Zhu, J.K.: A miRNA involved in phosphate-starvation response in Arabidopsis. Curr. Biol. 15: 2038-2043, 2005.

Gao, N., Qiang, X.M., Zhai, B.N., Min, J., Shi, W.M.: Transgenic tomato overexpressing ath-miR399d improves growth under abiotic stress conditions. - Russ. J. Plant Physiol. 62: 360-366, 2015.

Hackenberg, M., Shi, B.J., Gustafson, P., Langridge, P.: Characterization of phosphorus-regulated miR399 and miR827 and their isomirs in barley under phosphorussufficient and phosphorus-deficient conditions. - BMC Plant Biol. 13: 214, 2013.

Hai, B.Z., Qiu, Z.B., He, Y.Y., Yuan, M.M., Li, Y.F.: Characterization and primary functional analysis of Pinus densata miR171. - Biol. Plant. 62: 318-324, 2018.

Hu, B., Wang, W., Deng, K., Li, H., Zhang, Z., Zhang, L., Chu, C.C.: MicroRNA399 is involved in multiple nutrient starvation responses in rice. - Front. Plant Sci. 6: 188, 2015.

Lin, S.I., Chiang, S.F., Lin, W.Y., Chen, J.W., Tseng, C.Y., Chiou, W.T.: Regulatory network of microrna399 and pho2 by systemic signaling. - Plant Physiol. 147: 732-746, 2008.

Livak, K.J., Schmittgen, T.D.: Analysis of relative gene expression data using real-time quantitative PCR and the $2^{-\Delta \Lambda C t}$ method. - Methods 25: 402-408, 2001.

Qiu, Z.B., Hai, B.Z. Li, Y.F., Guo, J.L., Zhang, L.: Characterization of wheat miRNAs and their target genes responsive to cadmium stress. - Plant Physiol. Biochem. 101: 60-67, 2016.

Qiu, Z.B., Li, X.J., Zhao, Y.Y., Zhang, M.M., Wan, Y.L., Cao, D.C., Lu, S.F., Lin, J.X.: Genome-wide analysis reveals dynamic changes in microRNAs expression during vascular cambium development in Chinese fir (Cunninghamia lanceolata). - J. exp. Bot. 66: 3041-3054, 2015.

Qiu, Z.B., Wan, L.C., Chen, T., Wan, Y.L., He, X.Q., Lu, S.F., Wang, Y.W., Lin, J.X.: The regulation of cambial meristem activity in Chinese fir involves extensive remodelling of the transcriptome. - New Phytol. 199: 708-719, 2013.

Shi, J., Zhen, Y., Zheng, R.H.: Proteome profiling of early seed development in Cunninghamia lanceolata (Lamb.) Hook. - J. exp. Bot. 61: 2367-2381, 2010.

Voinnet, O.: Origin, biogenesis, and activity of plant microRNAs. - Cell 136: 669-687, 2009.

Wan, L.C., Wang, F., Guo, X.Q., Lu, S.F., Qiu, Z.B., Zhao, Y.Y., Zhang, H.Y., Lin, J.X.: Identification and characterization of small non-coding RNAs from Chinese fir by high throughput sequencing. - BMC Plant Biol. 12: 146, 2012.

Wang, Y., Zhang, J., Cui, W., Guan, C., Mao, W., Zhang, Z.: Improvement in fruit quality by overexpressing miR399a in woodland straEwberry. - J. Agr. Food Chem. 65: 7361-7370, 2017.

Zhang, Y.: miRU: an automated plant miRNA target prediction server. - Nucl. Acids Res. 33: W701-W704, 2005.

Zheng, Y., Li, Y.F., Sunkar, R., Zhang, W.: SeqTar: an effective method for identifying microRNA guided cleavage sites from degradome of polyadenylated transcripts in plants. - Nucl. Acids Res. 40: e28, 2012.

Zuker, M.: Mfold web server for nucleic acid folding and hybridization prediction. - Nucl. Acids Res. 13: 3406-3415, 2003. 